\title{
Rapid Detection of Mycobacterium tuberculosis by using MPT64 Immunochromatographic Assay
}

\author{
Dinesh Babu R*, Arun Kumar P, Heetandra Kumar Purohit and Shivashri C
}

Department of Microbiology, BKL Walawalkar Rural Medical College, Ratnagiri, Maharashtra, India

\begin{abstract}
Tuberculosis (TB) is one of the major infectious diseases in the developing countries and is more prevalent in India. The confirmatory identification of $M$. tuberculosis (MTB) is still being done by the conventional, laborious and time-consuming biochemical methods. Before this period of confirmation, the diseased patient is prone to encounter susceptible individuals; this clearly indicates the need for rapid, enhanced identification of Mycobacteria along with an effective drug sensitivity testing treatment. A specific antigen, MPT64 (major culture filtrate protein), differentiates the complex of $M$. tuberculosis from mycobacteria other than tuberculosis (MOTT) species. The present study evaluated the identification of Mycobacteria tuberculosis from MOTT by using SD Bioline kit and the findings were correlated with a conventional biochemical test and the sensitivity, specificity, and predictive values of this kit were also assessed. 80 $(n=80)$ mycobacterial isolates from Sputum and BAL were tested by using MPT64 immunochromatographic assay and validated by standard microscopic and biochemical assays. In the assay there was no significant difference observed in identifying MTBC isolates between SD MPT64 TB Ag Kit $囚$ assay and Biochemical test. Thus, SD Ag MPT64 kit displayed 100\% sensitivity, specificity, positive predictive and negative predictive values. Hence the kit is highly appropriate for use in TB diagnosis because of its simplicity, cost effectiveness and rapidity.
\end{abstract}

Keywords: Tuberculosis; Infectious diseases; Mycobacteria

\section{Introduction}

Tuberculosis (TB) caused by the infection of bacillus Mycobacterium tuberculosis (MTB) characteristically affects the lungs (pulmonary TB) but also may affect other sites (extra pulmonary TB). It is spread by air and potentially fatal disease of human [1]. The people infected by HIV are highly prone to develop TB disease [1]. It has been estimated by the World Health Organization (WHO) in 2013 that there about 9 million (range, 8.6 million-9.4 million) cases globally. There are about 2.0-2.3 million incident cases of TB annually reported in India, this accounts for a greater number than any other country (i.e.) a fifth of new cases in the world [1]. There is also an alarming increase in number of drug-resistant cases of TB. Therefore, the need for rapid and correct identification of Mycobacteria and rapid drug sensitivity testing for effective treatment of the disease is increasing $[2,3]$.

It is well known that though the automated culture systems Bactec 460, MBBacT (Bio Merieux, France), MGIT have reduced the time of culture, they do not differentiate MTB and MOTT. The conventional biochemical methods are being used for the identification of $M$. tuberculosis (MTB). These tests are time consuming and raise the need for safety precautions; during this process the infected individual spread the infection to many susceptible individuals. The current objective of WHO is to find rapid identification and detection of drug resistance within 2 days by using employing nucleic acid amplification assays. This needs a specialized set up and trained personal, which may not be affordable for poor countries [4]. The Standard Diagnostics, Inc. (SD) (Yongin, Korea) developed an MPT64-based, simple and rapid immunochromatographic assay known as the SD Bioline TB Ag MPT64 RAPID ${ }^{\infty}$ test (SD bioline kit). This lateral flow test has been shown to identify the M. tuberculosis complex from the MOTT, by impregnating the mouse monoclonal anti-MPT64 antibody on nitrocellulose membrane $[1,5,6]$.

The specific antigen MPT64 $(24 \mathrm{kDa})$ is one of the major culture filtrate protein encoded by RD2 in genes, which differentiates the M. tuberculosis complex from the mycobacteria other than tuberculosis
(MOTT) species $[7,8]$. The actively secreted proteins of M. tuberculosis are the first to interact with the host immune system and recognized by human Th1 cells. Therefore, it could be useful for TB diagnosis or as part of a novel candidate vaccine against TB $[9,10]$. A large amount of variability in the diagnostic accuracy of MPT64 has been reported, depending on the recombinant antigen used in assays $[7,8]$.

The present study used SD Bioline kit to evaluate the identification of MTB from MOTT and the findings were correlated with conventional biochemical test, and the sensitivity, specificity, and predictive values of this kit were also assessed.

\section{Materials and Methods}

$80(\mathrm{n}=80)$ mycobacterial isolates from Sputum and BAL, one control strain H3R7V, 15(n=15) Gram positive and gram-negative bacterial isolates from Urine, Sputum and BAL were tested by using MPT64 immunochromatographic assay and validated by standard microscopic and biochemical assays.

\section{Immunochromatographic (ICT) assay}

As per the manufacturer's instructions, MTB Antigen MPT64 rapid ICT kit (SD Bioline, Seoul, South Korea) was used [11]. The confirmation of MTB isolates was done by immobilizing the test kit with Mouse monoclonal antibodies, against MPT64 antigen, on a

*Corresponding author: Dinesh Babu R, Department of Microbiology, BKL Walawalkar Rural Medical College, Ratnagiri, Maharashtra-415606, India, Tel: 9994439903; E-mail: dinesh.shan14@gmail.com

Received July 12, 2018; Accepted August 06, 2018; Published August 13, 2018

Citation: Dinesh Babu R, Arun Kumar P, Purohit HK, Shivashri C (2018) Rapid Detection of Mycobacterium tuberculosis by using MPT64 Immunochromatographic Assay. J Med Microb Diagn 7: 282. doi:10.4172/2161-0703.1000282

Copyright: @ 2018 Dinesh Babu R, et al. This is an open-access article distributed under the terms of the Creative Commons Attribution License, which permits unrestricted use, distribution, and reproduction in any medium, provided the original author and source are credited. 
Citation: Dinesh Babu R, Arun Kumar P, Purohit HK, Shivashri C (2018) Rapid Detection of Mycobacterium tuberculosis by using MPT64 Immunochromatographic Assay. J Med Microb Diagn 7: 282. doi:10.4172/2161-0703.1000282

nitrocellulose membrane. The entire procedure was done inside a biosafety class II cabinet.

The 2-4 isolated colonies scraped from the solid LJ medium was suspended in $200 \mu \mathrm{l}$ of extraction buffer. Further this emulsified solution was applied in the sample well. After 15 minutes of incubation on sample application, the test was interpreted at room temperature. The entire procedure was carried out as per manufacturer instruction. The control region did not show any band, which was considered invalid. The test results were validated by biochemical test (Niacin test and Nitrate Reduction test).

\section{Results}

$80(\mathrm{n}=80)$ mycobacterial isolates were cultured in $\mathrm{LJ}$ media, after 8 weeks of incubation it was further confirmed by smear by using Ziel Neelson method. Validation of mycobacterial $s p p$ was done by niacin test and nitrate reduction test of these 80 isolates 75 were $(n=75)$ MTB and $5(\mathrm{n}=5)$ isolates were MOTT (Figure 1).

All 80 isolates showed pink color band in control line and One $\mathrm{H} 37 \mathrm{Rv}$ control strain shows pink colored band in both control and test line for confirming the presence of Tb MPT64 antigen. In the test line, 73 isolates of MTB displayed dark pink band, whereas 2 isolates showed faint band and were exposed to longer incubation period of 48 hours in the repeat test as well as biochemical test (niacin and nitrate reduction test) and was confirmed to be MTBC.

It was clearly observed that there was no significant difference shown in detection of MTBC isolates between SD MPT64 TB Ag Kit assay and Biochemical test. Hence, the sensitivity, specificity, positive predictive \& negative predictive values of the SD Ag MPT64 kit was found to be $100 \%$.

\section{Discussion}

One of the major health issues in developing countries is Tuberculosis (TB) and it has been shown that India stands for one-fifth of the total TB incident cases globally. The mortality rate in India was noted to be 2 persons every three minutes, nearly 1,000 every day [1].

The detection of acid-fast-bacilli by microscopic method is rapid but the sensitivity and the specificity to discriminate between

\section{Distribution of Mycobacterial Isolates}

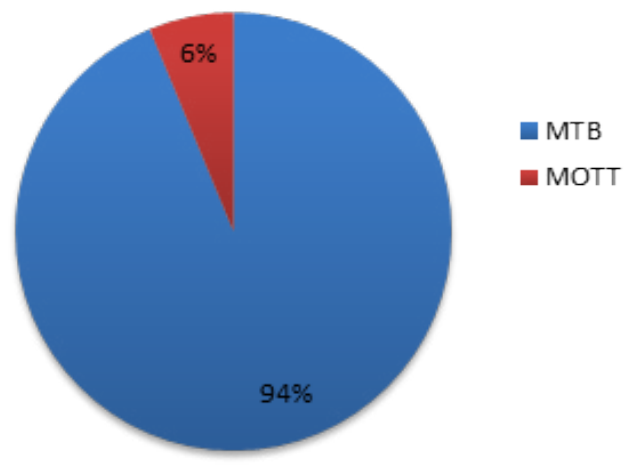

Figure 1: Distribution of mycobacterial isolates.
Mycobacterium spp. is low. The early diagnosis of TB is too difficult, and the well-equipped biosafety laboratory is required to carry out the culture, detection and drug vulnerability testing of MTB and it is vital for the management of TB incidence [1].

The rapid detection of the mycobacterial isolates using automated culture system has been highly recommended by Revised National Tuberculosis Control Program (RNTCP) yet there is a need for rapid detection method of MTB isolates. The newer rapid identification method has become the main concern in the diagnosis and patient management of tuberculosis [1]. The developing rapid ICT methods have known to be the ideal tool for the diagnosis of TB [12].

The main aim of the study is to evaluate rapid and economically cheaper test which detect accuracy of mycobacterial isolates. Differentiation of MTB from MOTT is very important for clinically and therapeutically. In most of the laboratories using conventional biochemical and smear microscopy for differentiation. It requires skilled labor and time-consuming process for identification. But these new immunochromatographic tests doesn't not require skilled labor and require biosafety cabinet to perform, to get accuracy of the results within 20 minutes to confirm. 784 culture isolates for rapid identification of MTB was evaluated by the commercially available ICT test kit, and Accu probe -MTB was used as the reference method for comparative evaluation. The rapid test kit displayed sensitivity of about $99.2 \%$ (381/384) and it showed no false positive results. Thus, this clearly demonstrates that the ICT test showed $100 \%$ specificity, which could be a useful method in the routine rapid identification of MTB isolate [13].

Few studies have evaluated by using SD MPT64 TB antigen rapid ICT kits, with sensitivity ranging from 97 to $100 \%$ and a specificity of $100 \%$ and false negative results occurs due to unique mutations in the MPT64 gene have been reported [14-16]. This is a common feature encountered with both commercial kits.

\section{Conclusion}

In this study, there was no significant difference found in the predictive values, sensitivity and specificity for isolates. The band intensity was more prominent in the liquid cultures. The study attempted to evaluate the ICT for the detection of MPT64 antigen in smear positive sputum samples. The total expenditure of ICT and biochemical tests will be approximately same.

The swiftness of the test will markedly reduce the time of MTB culture and DST; thus, it can significantly contribute in TB control program. Our study results clearly demonstrated the use of SD Bioline test for the mycobacteria identification. The conventional identification tests can be effectively replaced by the immunochromatographic assays for detecting MP64 antigen. Thus, this rapid, simple and cost-effective method can be used in TB diagnostic laboratories.

\section{References}

1. WHO (2014) Global tuberculosis report 2014.

2. Tomiyama T, Matsuo K, Abe C (1997) Rapid identification of Mycobacterium tuberculosis by an immunochromatography using anti MPB64 monoclonal antibodies. Int J Tuberc Lung Dis 1: 59.

3. WHO (2013) HIV-Associated TB Facts 2013.

4. Arora J, Kumar G, Verma AK, Bhalla M, Sarin R, et al. (2015) Utility of MPT64 antigen detection for rapid confirmation of Mycobacterium tuberculosis complex. J glob infec disease 7: 66-69.

5. Ang CF, Cajucom MA, Kim Y, Bang H, Lee H, et al. (2011) Evaluation of a rapid assay for identification of Mycobacterium tuberculosis grown in solid and liquid media. Int J Tuberc Lung Dis 15: 1475-147. 
Citation: Dinesh Babu R, Arun Kumar P, Purohit HK, Shivashri C (2018) Rapid Detection of Mycobacterium tuberculosis by using MPT64 Immunochromatographic Assay. J Med Microb Diagn 7: 282. doi:10.4172/2161-0703.1000282

6. Maurya AK, Nag VL, Kant S, Kushwaha RA, Kumar M, et al. (2012) Evaluation of an immunochromatographic test for discrimination between mycobacterium tuberculosis complex and non-tuberculous mycobacteria in clinical isolates from extra-pulmonary tuberculosis. Indian J Med Res 135: 901-196.

7. Park MY, Kim YJ, Hwang SH, Kim HH, Lee EY, et al. (2009) Evaluation of an immunochromatographic assay kit for rapid identification of Mycobacterium tuberculosis complex in clinical isolates. J Clin Microbiol 47: 481-484.

8. Silva VM, Sardella IG, Luiz RR, Cunha AJ, Cavalcanti AH, et al. (2008) Immunoreactivity of five antigens of Mycobacterium tuberculosis in patients attending a public health care facility in an area with high endemicity for TB. Microbiol Immunol 52: 544-550.

9. Yang H, Liu ZH, Zhang LT, Wang J, Yang HS, et al. (2011) Selection and application of peptide mimotopes of MPT64 protein in Mycobacterium tuberculosis. J Med Microbiol 60: 69-74.

10. Mustafa AS (2009) HLA-promiscuous Th1-cell reactivity of MPT64 (Rv1980c), a major secreted antigen of Mycobacterium tuberculosis, in healthy subjects. Med Princ Pract 18: 385-392.
11. Mustafa AS, Shaban F (2010) Mapping of Th1-cell epitope regions of Mycobacterium tuberculosis protein MPT64 (Rv1980c) using synthetic peptides and T-cell lines from M. tuberculosis-infected healthy humans. Med Princ Pract 19: $122-112$.

12. http://www.standardia.com/en/home/product/rapid/infectious-disease/TB_Ag_ MPT64.html.

13. Kumar VGS, Urs TA, Ranganath RR (2011) MPT 64 Antigen detection for rapid confirmation of $M$. tuberculosis isolates. BMC Res Notes 4: 79-82.

14. Shen $\mathrm{GH}$, Chen $\mathrm{CH}$, Hung $\mathrm{CH}$, Wu KM, Lin CF (2009) Combining the Capilia TB assay with smear morphology for the identification of Mycobacterium tuberculosis complex. Int J Tuberc Lung Dis 13: 371-376.

15. Gaillard T, Fabre M, Martinaud C, Vong R, Brisou P, et al. (2011) Assessment of the SD Bioline Ag MPT64 Rapid ${ }^{\mathrm{TM}}$ and the MGITTM TBc identification tests for the diagnosis of tuberculosis. Diagn Microbiol Infect Dis 70: 154-156.

16. Hirano K, Aono A, Takahashi M, Abe C (2004) Mutations including IS6110 insertion in the gene encoding the MPT64 protein of Capilla TB-negative Mycobacterium tuberculosis isolates. J Clin Microbiol 42: 390-392. 